OPEN ACCESS

Edited by:

Alberto Porta,

University of Milan, Italy

Reviewed by:

Antonio Roberto Zamunér,

Catholic University of Maule, Chile

Francesca Perego,

Istituti Clinici Scientifici Maugeri (ICS

Maugeri), Italy

*Correspondence:

Cheng Wang

wangcheng2nd@csu.edu.cn orcid.org/0000-0002-7120-0654

Specialty section:

This article was submitted to

Autonomic Neuroscience,

a section of the journal

Frontiers in Neuroscience

Received: 22 May 2021

Accepted: 23 July 2021

Published: 12 August 2021

Citation:

Zou R, Wang S, Cai H, Li F, Lin P, Wang $Y$ and Wang C (2021) Vitamin D Deficiency in Children With Vasovagal Syncope Is Associated With Impaired Circadian Rhythm of Blood Pressure.

Front. Neurosci. 15:712462.

doi: 10.3389/fnins.2021.712462

\section{Vitamin D Deficiency in Children With Vasovagal Syncope Is Associated With Impaired Circadian Rhythm of Blood Pressure}

\author{
Runmei Zou' ${ }^{1}$, Shuo Wang ${ }^{2}$, Hong Cai ${ }^{1}$, Fang Li', Ping Lin' ${ }^{1}$, Yuwen Wang ${ }^{1}$ and \\ Cheng Wang ${ }^{1 *}$
}

' Department of Pediatric Cardiovasology, Children's Medical Center, The Second Xiangya Hospital, Central South University, Changsha, China, ${ }^{2}$ Department of Pediatrics, Xiangya Hospital, Central South University, Changsha, China

Background: Vitamin D deficiency is associated with the risk of cardiovascular diseases. We aimed to investigate the serum vitamin $D$ levels in children with vasovagal syncope (WS) and explore the correlation of vitamin D status and circadian rhythm of blood pressure in WS pediatric patients.

Methods: This was a retrospective study. 130 syncopal children diagnosed with WS were included in the study. 110 age and gender matched healthy individuals were enrolled as control. According to serum 25(OH)D levels, WS patients were divided into vitamin D sufficient group and vitamin D deficient group. Detailed information of WS children with vitamin D deficiency and sufficiency on demographic data, baseline laboratory testing, echocardiogram, ambulatory blood pressure monitoring, and Holter ECG recording were extracted and analyzed.

Results: WS children had a higher prevalence of vitamin D deficiency compared with healthy individuals (33.8\% vs. 20.0\%, $P=0.017)$. WS patients with vitamin D deficiency had a higher rate of non-dipper blood pressure $(79.5 \%$ vs. $59.3 \%, P=0.021)$ and a lower value of square root of mean squared differences of successive normal to normal intervals (rMSSD) (median 107.8 vs. $141.0 \mathrm{~ms}, P=0.035$ ) compared with those with vitamin D sufficiency. Logistic regression analysis showed that non-dipper blood pressure was associated with serum 25(OH)D level [OR $=0.979,95 \% \mathrm{Cl}(0.960,0.999)$, $P=0.036]$.

Conclusion: WS pediatric patients had a higher prevalence of vitamin D deficiency. WS children with vitamin $D$ deficiency showed a higher rate of non-dipper blood pressure, suggesting that vitamin D deficiency is correlated with impaired circadian rhythm of blood pressure.

Keywords: ambulatory blood pressure monitoring, vasovagal syncope, vitamin D, children, circadian rhythm

\section{INTRODUCTION}

Vasovagal syncope (VVS), the most common cause of syncope in pediatric patients (Villafane et al., 2021), presents with an inability to maintain postural tone and very brief loss of consciousness, followed by spontaneous recovery without neurologic sequelae. VVS is related to emotional stress, prolonged standing, postural changes, pain, and a crowded environment. The mechanism of VVS 
remains incompletely revealed. Excessive activation of vagal tone via dysregulation of Bezold-Jarisch reflex, together with a decreased sympathetic tone, contributes to the pathogenesis of VVS (Stewart et al., 2017). It is thought that VVS is a benign condition and does not lead to mortality so far. However, frequent syncope increases the risk of injuries and reduces the quality of life significantly (Grimaldi Capitello et al., 2016).

Vitamin D is a group of fat-soluble molecules, playing a vital role in regulating numerous physiological processes in addition to calcium and phosphorus homeostasis. Though the effect of vitamin D deficiency and cardiovascular disease is controversial, observational studies reported an association between vitamin $\mathrm{D}$ deficiency and the risk of cardiovascular disease (Latic and Erben, 2020; Xie et al., 2020). Adolescents suffering from severe fatigue and/or orthostatic intolerance exhibited vitamin $\mathrm{D}$ deficiency (Antiel et al., 2011). The prevalence of hypovitaminosis D (25hydroxyvitamin $\mathrm{D} \leq 50 \mathrm{nmol} / \mathrm{L}$ ) was $22 \%$ in adolescent patients with excessive postural tachycardia, while it was $14 \%$ in healthy individuals (Saintonge et al., 2009; Antiel et al., 2011). A previous study reported that syncopal adult patients had a lower level of vitamin D when compared to healthy individuals, especially for VVS patients diagnosed by head-up tilt test (HUTT) (Usalp et al., 2020). For pediatric patients with VVS, vitamin D levels were significantly decreased compared with healthy controls, and correlation analysis suggested that serum 25-hydroxyvitamin D levels may be associated with rMSSD values (Zhang et al., 2021). These results indicated an important role of vitamin D in VVS. However, data on the correlation between serum vitamin $\mathrm{D}$ level and VVS in pediatric patients need to be further explored.

In the study, we aimed to investigate the vitamin $\mathrm{D}$ level in pediatric patients with VVS and compare the clinical characteristics of VVS pediatric patients with vitamin D sufficiency and vitamin D deficiency to explore the correlation of vitamin D status and circadian rhythm of blood pressure.

\section{STUDY POPULATION AND METHODS}

\section{Study Population and Data Collection}

This was a retrospective study and data were collected from patients who complained of recurrent syncope and visited Syncope Ward, Children's Medical Center, The Second Xiangya Hospital, Central South University between January 2018 and December 2019. The sample sizes was calculated according to the following formula: $n_{1}=n_{2}=2\left[\left(\mathrm{t}_{\alpha}+\mathrm{t}_{\beta}\right) \mathrm{s} / \delta\right]^{2}[\alpha=0.05$ (twoside), $\beta=0.10, s=25, \delta=10]$. Two researchers who were blinded to the study protocol collected the data separately. Patients with neurological, cardiogenic, and psychological diseases were excluded. 130 syncopal patients (4-15 years old) diagnosed with VVS by HUTT were recruited in the study. Detailed information of VVS patients on demographic data, baseline laboratory testing, echocardiogram, ambulatory blood pressure monitoring, and Holter ECG recording were reviewed and extracted. 110 age and gender matched healthy individuals (4-16 years old) without malnutrition were enrolled as control. These children came to visit our child health clinic for physical examination between January 2018 and December 2019. VVS patients were divided into two groups as those with vitamin $\mathrm{D}$ levels above or below $50 \mathrm{nmol} / \mathrm{L}$ (Holick et al., 2011).

\section{Methods HUTT Protocol}

The protocol of HUTT had been conducted according to the previous study (Wang et al., 2018). The HUTT was approved by the Ethics Committee of The Second Xiangya Hospital, Central South University [Ethical Audit No. Study 012 (2014)]. Informed consent was issued by all the subjects directly or their guardians prior to the test. Subjects were asked to lay still for $10 \mathrm{~min}$, and then basic heart rate (HR), blood pressure (BP), and ECG were recorded. Subjects were tilted at $60^{\circ}$ head upward. HR, $\mathrm{BP}$, and ECG were recorded continuously until either $45 \mathrm{~min}$ duration or development of syncope or intolerable near syncope symptoms. If syncope occurred, subjects were rapidly put in the supine position. If syncope or presyncope did not occur, tilted posture was maintained, subjects were sublingually medicated with nitroglycerin $(4-6 \mu \mathrm{g} / \mathrm{kg}$, maximum $\leq 300 \mu \mathrm{g})$, and $\mathrm{HR}$, $\mathrm{BP}$, and ECG were recorded until for $20 \mathrm{~min}$ or syncope or presyncope occurred.

Vasovagal syncope was defined as the development of syncope or presyncope accompanied by hypotension (systolic $\mathrm{BP} \leq 80 \mathrm{mmHg}$ in children, and/or diastolic $\mathrm{BP} \leq 50 \mathrm{mmHg}$, or over $25 \%$ decrease in mean $\mathrm{BP}$ ), bradycardia $(<75 \mathrm{bpm}$ in children between 4 and 6 years old, $<65 \mathrm{bpm}$ in children between 6 and 8 years old, and $<60 \mathrm{bpm}$ in children above 8 years old), or cardiac arrest $>3$ s (Wang et al., 2018).

\section{Ambulatory Blood Pressure Monitoring (ABPM)}

Twenty-four-hour BP data were obtained from a 24-h ABPM device with a suitably sized cuff (ABPM6100, Welch Allyn, United States). The subjects were asked to continue their regular activity and sleep schedule, but to stay motionless during the period of BP measurement. BP data were measured every $15 \mathrm{~min}$ at daytime and every $30 \mathrm{~min}$ at nighttime. Daytime BP was defined as the mean of BP values measured from 6:00 to 22:00 h, whereas nocturnal BP was defined as the mean of BP values measured from 22:00 to 6:00 h of the next day. For acquiring high-quality $\mathrm{ABPM}$ recordings, $\mathrm{ABPM}$ data should meet the following conditions: at least 70\% valid recordings, at least 20 daytime and 7 nighttime valid recordings, and at least one valid recording for each hour. The nocturnal $\mathrm{BP}$ dipping rate was defined based on systolic blood pressure (SBP) data as follows: (daytime SBP-nighttime SBP)/(daytime SBP) $\times 100$. Nocturnal dipping status was classified into two categories according to the dipping rate in the study, dipper $(\geq 10 \%$ but $<20 \%)$ and non-dipper $(\geq 0 \%$ but $<10 \%$ ) (Williams et al., 2018).

\section{Heart Rate Variability (HRV)}

Twenty-four-hour electrocardiogram was recorded by 12channel 24 h Holter devices (TLC 4000 Dynamic ECG Recording Analyzer, Kangtai, China). The sampling rate was $10,000 \mathrm{~Hz}$ and the response band 0.05 to $60 \mathrm{HZ}$. HRV was analyzed by using an automatic Holter analysis system (TLC4000, Kangtai, China). Each RR interval was manually screened before the analysis. Only normal to normal (NN) beats were used for analysis with 
intervals. Interfering signals were automatically excluded by the standardized QRS peak detection of the analysis system. The time domain parameters, including standard deviations for all NN heart rate intervals over $24 \mathrm{~h}$ (SDNN), the standard deviation of the averages of NN intervals calculated over 5-min periods of the entire recording (SDANN), percentage of differences between adjacent RR intervals that are $>50 \mathrm{~ms}$ (PNN50), and square root of mean squared differences of successive NN intervals (rMSSD) were analyzed (Wang et al., 2019).

\section{Statistical Analysis}

Statistical analysis was performed by SPSS 24.0 (IBM Corp., Armonk, NY, United States). Continuous variables for data following normal distribution were described as mean \pm SD and analyzed by Student $t$-tests. Continuous variables for data not following normal distribution were expressed as the median with interquartile range (IQR) and analyzed using the Mann-Whitney $U$ test. Categorical data were described using frequencies and percentages. The $\chi^{2}$ test or Fisher's exact test was used to compare the differences of the categorical variables. Association for serum 25(OH)D level and rMSSD was analyzed by Spearman correlation coefficient. For the logistic regression model, variables including age, gender, body mass index, serum 25(OH)D level, and rMSSD that were associated with non-dipper BP were enrolled. $P$-value $<0.05$ was considered to be statistically significant differences.

\section{RESULTS}

A total of 130 VVS patients [mean age $10.7 \pm 2.5$ years, 69 females (53.1\%)] and 110 healthy individuals [mean age $10.3 \pm 2.3$ years, 50 females $(45.5 \%)]$ were included in the study. There were no significant differences in age, gender, and body mass index (BMI) between healthy individuals and VVS patients (all $P>0.05$ ). Serum 25(OH)D level was significantly lower in patients with VVS compared with healthy individuals $(59.8 \pm 21.4$ vs. $65.9 \pm 19.2 \mathrm{nmol} / \mathrm{L}, P=0.022)$. VVS patients had a higher rate of vitamin $\mathrm{D}$ deficiency compared with healthy individuals (33.8\% vs. $20.0 \%, P=0.017$ ) (Table $\mathbf{1}$ ).

According to serum vitamin D level, VVS patients were divided into two groups, vitamin $\mathrm{D}$ sufficient group with serum

TABLE 1 | Clinical characteristics of healthy individuals and vasovagal syncope patients.

\begin{tabular}{lccc}
\hline Variables & Control group $(\boldsymbol{n}=\mathbf{1 1 0})$ & VVS group $(\boldsymbol{n}=\mathbf{1 3 0})$ & $\boldsymbol{P}$ \\
\hline Male, $n(\%)$ & $60(54.5)$ & $61(46.9)$ & 0.239 \\
Female, $n(\%)$ & $50(45.5)$ & $69(53.1)$ & \\
Age (years) & $10.3 \pm 2.3$ & $10.7 \pm 2.5$ & 0.143 \\
BMl (kg/m²) & $17.8 \pm 4.1$ & $17.3 \pm 2.9$ & 0.290 \\
Serum 25(OH)D & $65.9 \pm 19.2$ & $59.8 \pm 21.4$ & 0.022 \\
(nmol/L) & & & \\
25(OH)D & $22(20.0)$ & & \\
deficiency rate, & & & \\
$n(\%)$ & & & \\
\hline
\end{tabular}

BMI, body mass index.
$25(\mathrm{OH}) \mathrm{D}$ level greater than or equal to $50 \mathrm{nmol} / \mathrm{L}$ and vitamin $\mathrm{D}$ deficient group with serum $25(\mathrm{OH}) \mathrm{D}$ level below $50 \mathrm{nmol} / \mathrm{L}$. As shown in Table 2, There was no significant difference between the two groups regarding gender, age, BMI, and laboratory findings including white blood counts, hemoglobin, platelets, serum thyroid-stimulating hormone, glucose, alanine aminotransferase, aspartate aminotransferase, serum creatinine, and uric acid (all $P>0.05)$.

There was no significant difference between VVS patients with sufficient and deficient vitamin $\mathrm{D}$ when compared on ejection fraction, fraction shortening, daytime and nocturnal systolic blood pressure, daytime and nocturnal diastolic blood pressure, and mean heart rate (all $P>0.05$ ). Parameters of HRV, SDNN, SDANN, and PNN50 had no significant differences between the two groups. However, rMSSD of VVS children with vitamin $\mathrm{D}$ deficiency was lower than those with vitamin $\mathrm{D}$ sufficiency (median 107.8 vs. $141.0 \mathrm{~ms}, P=0.035$ ) (Table 3). Spearman correlation analysis demonstrated that rMSSD was not correlated with 25(OH)D levels $(r=0.166, P=0.072)$. VVS patients with vitamin $\mathrm{D}$ deficiency had a higher rate of non-dipper blood pressure compared with those with normal vitamin D levels (79.5\% vs. 59.3\%, $P=0.021$ ) (Table 3). Serum 25(OH)D level, rMSSD, age, gender, and BMI were enrolled for logistic regression analysis, results showed that non-dipper blood pressure was associated with serum $25(\mathrm{OH}) \mathrm{D}$ level [OR $=0.979,95 \% \mathrm{CI}(0.960$, 0.999), $P=0.036$ ], but not related to $\mathrm{rMSSD}$, age, gender, and BMI (all $P>0.05$ ) (Table 4).

\section{DISCUSSION}

In the present study, we found that serum 25(OH)D level was lower in VVS patients than that of healthy individuals. VVS patients had a higher prevalence of vitamin deficiency. VVS

TABLE 2 | Demographic characteristics and laboratory findings of patients with vasovagal syncope.

\begin{tabular}{|c|c|c|c|}
\hline Variables & $\begin{array}{c}25(\mathrm{OH}) \mathrm{D} \\
\text { sufficient } \\
\text { group }(n=86)\end{array}$ & $\begin{array}{c}25(\mathrm{OH}) \mathrm{D} \\
\text { deficient } \\
\text { group }(n=44)\end{array}$ & $P$-value \\
\hline Male, $n(\%)$ & $44(51.2)$ & $17(38.6)$ & 0.176 \\
\hline Female, $n(\%)$ & $42(48.8)$ & $27(61.4)$ & \\
\hline Age (years) & $10.7 \pm 2.5$ & $10.7 \pm 2.4$ & 0.971 \\
\hline BMI $\left(\mathrm{kg} / \mathrm{m}^{2}\right)$ & $17.3 \pm 2.5$ & $17.2 \pm 3.5$ & 0.855 \\
\hline WBC $\left(\times 10^{9} / \mathrm{L}\right)$ & $6.1 \pm 1.5$ & $5.58 \pm 0.9$ & 0.081 \\
\hline HGB (g/L) & $126.1 \pm 12.5$ & $122.8 \pm 24.8$ & 0.415 \\
\hline $\operatorname{PLT}\left(\times 10^{9} / \mathrm{L}\right)$ & $278.0 \pm 65.1$ & $279.5 \pm 64.0$ & 0.917 \\
\hline TSH (ulU/ml) & $2.8 \pm 1.4$ & $3.2 \pm 1.8$ & 0.469 \\
\hline Glucose (mmol/L) & $4.5 \pm 0.7$ & $4.5 \pm 0.6$ & 0.978 \\
\hline$A L T, M(\mid Q R), U / L$ & $10.4(8.7,14.4)$ & $10.7(7.1,12.7)$ & 0.487 \\
\hline AST, M(IQR), U/L & $\begin{array}{c}21.5(17.3 \\
25.7)\end{array}$ & $\begin{array}{c}20.3(15.4 \\
26.5)\end{array}$ & 0.207 \\
\hline $\mathrm{SCr}(\mu \mathrm{mol} / \mathrm{L})$ & $41.7 \pm 10.3$ & $43.7 \pm 9.6$ & 0.422 \\
\hline UA ( $\mu \mathrm{mol} / \mathrm{L})$ & $301.4 \pm 81.6$ & $299.9 \pm 87.3$ & 0.936 \\
\hline
\end{tabular}

BMI, body mass index; WBC, white blood cell; HGB, hemoglobin; PLT, platelet; $T S H$, thyroid stimulant hormone; ALT, alanine aminotransferase; AST, aspartate aminotransferase; SCr, serum creatinine; UA, uric acid; IQR, inter quartile range. 
TABLE 3 | Electro-cardiological indictors of patients with vasovagal syncope.

\begin{tabular}{|c|c|c|c|}
\hline Variables & $\begin{array}{c}25(\mathrm{OH}) \mathrm{D} \\
\text { sufficient } \\
\text { group }(n=86)\end{array}$ & $\begin{array}{c}\text { 25(OH)D } \\
\text { deficient } \\
\text { group }(n=44)\end{array}$ & $\boldsymbol{P}$ \\
\hline EF (\%) & $66.6 \pm 3.7$ & $66.6 \pm 3.8$ & 0.987 \\
\hline FS (\%) & $36.2 \pm 4.0$ & $36.8 \pm 3.3$ & 0.455 \\
\hline dSBP (mmHg) & $107.3 \pm 16.4$ & $103.9 \pm 23.4$ & 0.354 \\
\hline $\mathrm{dDBP}(\mathrm{mmHg})$ & $60.7 \pm 6.7$ & $60.6 \pm 5.0$ & 0.965 \\
\hline nSBP (mmHg) & $98.8 \pm 15.8$ & $100.7 \pm 10.4$ & 0.489 \\
\hline nDBP (mmHg) & $51.8 \pm 6.6$ & $53.0 \pm 5.0$ & 0.305 \\
\hline Non-dipper BP, $n$ (\%) & $51(59.3)$ & 35 (79.5) & 0.021 \\
\hline mHR (beats/min) & $80.5 \pm 8.3$ & $79.4 \pm 9.4$ & 0.538 \\
\hline SDNN, M(IQR), ms & $\begin{array}{c}164.2(141.3 \\
191.6)\end{array}$ & $\begin{array}{c}153.0(133.5 \\
189.7)\end{array}$ & 0.248 \\
\hline SDANN (ms) & $\begin{array}{c}146.5(122.1 \\
181.9)\end{array}$ & $\begin{array}{c}138.1(109.8 \\
171.9)\end{array}$ & 0.266 \\
\hline rMSSD, M(IQR), ms & $\begin{array}{c}141.0(96.5 \\
193.7)\end{array}$ & $\begin{array}{c}107.8(79.8 \\
146.5)\end{array}$ & 0.035 \\
\hline PNN50 (\%) & $38.1 \pm 15.3$ & $35.1 \pm 15.6$ & 0.330 \\
\hline
\end{tabular}

EF, ejection fraction; FS, fraction shortening; dSBP, daytime systolic blood pressure; dDBP, daytime diastolic blood pressure; $n S B P$, nocturnal systolic blood pressure; $n D B P$, nocturnal diastolic blood pressure (Daytime BP was defined as the mean of $B P$ values measured from 6:00 to 22:00 h, whereas nocturnal $B P$ was defined as the mean of BP values measured from 22:00 to 6:00 $\mathrm{h}$ of the next day); $m H R$, mean heart rate; SDNN, standard deviations for all normal to normal (NN) heart rate intervals over $24 h$; SDANN, standard deviation of the averages of NN intervals calculated over 5-min periods of the entire recording; $r M S S D$, square root of mean squared differences of successive NN intervals; PNN50, percentage of differences between adjacent RR intervals that are >50 ms; $M$, median; IQR, inter quartile range.

TABLE 4 | Logistic regression analysis between non-dipper blood pressure and serum 25(OH)D level, rMSSD, gender, age, and BMI.

\begin{tabular}{lrccccc}
\hline Variables & Beta & SE & Ward & P-value & OR & 95\% Cl of OR \\
\hline 25(OH)D level & -0.021 & 0.010 & 4.408 & 0.036 & 0.979 & $0.960,0.999$ \\
rMSSD & 0.004 & 0.003 & 1.762 & 0.184 & 1.004 & $0.998,1.009$ \\
Gender & -0.125 & 0.409 & 0.094 & 0.760 & 0.882 & $0.395,1.968$ \\
Age & -0.090 & 0.093 & 0.947 & 0.330 & 0.914 & $0.762,1.096$ \\
BMl & -0.082 & 0.078 & 1.097 & 0.295 & 0.921 & $0.790,1.074$ \\
\hline
\end{tabular}

BMI, body mass index; rMSSD, square root of mean squared differences of successive normal to normal intervals.

patients with vitamin $\mathrm{D}$ deficiency exhibited a higher rate of nondipper blood pressure compared with those with normal vitamin D levels. Logistic regression analysis suggested that non-dipper blood pressure was closely associated with serum vitamin D level.

Vasovagal syncope is caused by an abnormal reaction of the autonomic system to various stimuli, such as a triggering event or posture change. Prolonged standing or posture change leads to decreased venous return, which results in inadequate ventricular filling and vigorous cardiac contraction. Mechanoreceptors (C fibers) located preferentially in the inferolateral wall of the left ventricle are activated, resulting in hypotension and paradoxical bradycardia due to increased activity of inhibitory receptors and consequent parasympathetic hyperactivity (Medow et al., 2008).

In our study, VVS patients exhibited a higher rate of vitamin $\mathrm{D}$ deficiency than healthy individuals. The reason why VVS patients had vitamin D deficiency may be concluded in several mechanisms. Firstly, vitamin D is important in maintaining cardiac autonomic function. The low level of vitamin D $(<50 \mathrm{nmol} / \mathrm{L})$ caused cardiac autonomic dysfunction by repressing vagal activity and increased the risk of cardiovascular diseases (Mann et al., 2013). Vitamin D supplementation significantly improved HRV parameters of healthy individuals with vitamin D deficiency (Mann et al., 2014; Dogdus et al., 2019). Secondly, the active form of vitamin D plays a critical role in the proliferation of vascular smooth muscle cells and endothelial cells. Vitamin D regulates blood pressure by acting on vascular smooth muscle cells and endothelial cells (de la Guia-Galipienso et al., 2021). Endothelium-derived nitric oxide-evoked dilation was decreased in arteries from vitamin $\mathrm{D}$ deficient rats (Tare et al., 2011), suggesting vitamin D deficiency prompts endothelial vasodilator dysfunction. Vitamin D deficiency may result in syncope by deterioration of vascular function. Moreover, vitamin $\mathrm{D}$ deficiency may disturb neuronal conduction in the baroreflex mechanism (Naveilhan et al., 1996), which is closely related to the onset of syncope.

During the course of $24 \mathrm{~h}, \mathrm{BP}$ behavior exhibits a trend characterized by lower BP at nighttime than at daytime. For healthy individuals, BP at nighttime decreases at $10-20 \%$ and with a peak in the early morning. Previous studies indicated that abnormal diurnal blood pressure profile is associated with subclinical target organ damage, cardiovascular events, and mortality (Gong et al., 2019). In a previous study of newly diagnosed hypertension, patients with vitamin $\mathrm{D}$ deficiency had a higher nighttime SBP and non-dipper SBP pattern compared to patients with sufficient vitamin D (Cakal et al., 2021). In our research, there was no difference in daytime and nocturnal BP between VVS children with sufficient and deficient vitamin D. However, children with vitamin D deficiency had a higher rate of non-dipper blood pressure. We further performed logistic regression analysis, and the results showed that non-dipper blood pressure was associated with serum vitamin D levels. Vitamin D is important in maintaining cardiac autonomic function. Vitamin $\mathrm{D}$ deficiency may disturb autonomic function, resulting in the disorder of blood pressure circadian rhythm.

Heart rate variability is a non-invasive and simple method for the assessment of cardiac autonomic function (Lahiri et al., 2008). It consists of time-domain and frequency-domain analysis. In the time-domain analysis, SDNN indicates the general measurement of autonomic nervous system balance, rMSSD and PNN50 indicate the parasympathetic activity of heart. In the frequency-domain analysis, HF is under the influence of the parasympathetic nervous system, while LF is modulated by both parasympathetic and sympathetic nervous systems. Previous studies demonstrated that HRV parameters as SDNN, SDANN, rMSSD, PNN50, and HF were significantly decreased in healthy individuals with vitamin $\mathrm{D}$ deficiency compared with those with sufficient vitamin D (Canpolat et al., 2015; Dogdus et al., 2019). These results suggested that vitamin D deficiency was significantly associated with impaired cardiac autonomic functions in healthy individuals. In children and adolescents with VVS, vitamin D deficiency exhibited a lower rMSDD value compared to the vitamin 
D sufficient group (Zhang et al., 2021). In our study, rMSDD value was decreased in VVS children with vitamin $\mathrm{D}$ deficiency compared to those with vitamin D sufficiency, which was consistent with the previous study. The result demonstrates that vitamin $\mathrm{D}$ deficiency may be related to the decreased parasympathetic activity of the heart, paradoxically activates sympathetic activity, which makes the patients more prone to trigger the Bezold-Jarisch reflex and suffer syncope. However, Spearman analysis indicated that rMSSD value was not correlated with serum vitamin D levels in our study. More studies should be performed to explore the relation between vitamin $\mathrm{D}$ levels and HRV in VVS children.

Our study is a retrospective study, and there are some limitations. First, vitamin D is regulated by calcium, phosphate, fibroblast growth factor 23 (FGF23), and parathyroid hormone (PTH) levels. However, data of calcium, phosphate, FGF23 and PTH levels were not tested in the study. Besides, data on ambulatory blood pressure for healthy individuals were not obtained in our study. The circadian rhythm of blood pressure for healthy individuals with vitamin D deficiency was not analyzed.

In spite of its limitation, the findings of our research showed that VVS pediatric patients had a higher prevalence of vitamin D deficiency compared with healthy children. VVS pediatric patients with vitamin $\mathrm{D}$ deficiency showed a higher rate of non-dipper blood pressure, suggesting little blood pressure variability in these patients. Arterial blood pressure variability could detect early autonomic alterations (Genovesi et al., 2008). Analysis of arterial blood pressure variability may be helpful for further assessing autonomic function of VVS children with vitamin D deficiency. Furthermore, vitamin $\mathrm{D}$ deficiency may be correlated with impaired circadian rhythm of blood pressure in VVS pediatric patients. Whether vitamin D supplementation is helpful to improve the circadian rhythm of blood pressure and autonomic function should be further explored.

\section{REFERENCES}

Antiel, R. M., Caudill, J. S., Burkhardt, B. E., Brands, C. K., and Fischer, P. R. (2011). Iron insufficiency and hypovitaminosis D in adolescents with chronic fatigue and orthostatic intolerance. South. Med. J. 104, 609-611. doi: 10.1097/ SMJ.0b013e3182246809

Cakal, S., Cakal, B., and Karaca, O. (2021). Association of vitamin D deficiency with arterial stiffness in newly diagnosed hypertension. Blood Press. Monit. 26, 113-117. doi: 10.1097/MBP.0000000000000497

Canpolat, U., Ozcan, F., Ozeke, O., Turak, O., Yayla, C., Acikgoz, S. K., et al. (2015). Impaired cardiac autonomic functions in apparently healthy subjects with vitamin D deficiency. Ann. Noninvasive Electrocardiol. 20, 378-385. doi: 10.1111/anec. 12233

de la Guia-Galipienso, F., Martinez-Ferran, M., Vallecillo, N., Lavie, C. J., SanchisGomar, F., and Pareja-Galeano, H. (2021). Vitamin D and cardiovascular health. Clin. Nutr. 40, 2946-2957. doi: 10.1016/j.clnu.2020.12.025

Dogdus, M., Burhan, S., Bozgun, Z., Cinier, G., Koyuncu, I., Yucel, et al. (2019). Cardiac autonomic dysfunctions are recovered with vitamin D replacement in apparently healthy individuals with vitamin D deficiency. Ann. Noninvasive. Electrocardiol. 24:e12677. doi: 10.1111/anec.12677

Genovesi, S., Pieruzzi, F., Giussani, M., Tono, V., Stella, A., Porta, A., et al. (2008). Analysis of heart period and arterial pressure variability in childhood hypertension: key role of baroreflex impairment. Hypertension 51, 1289-1294. doi: 10.1161/HYPERTENSIONAHA.107.109389

\section{DATA AVAILABILITY STATEMENT}

The raw data supporting the conclusions of this article will be made available by the authors, without undue reservation.

\section{ETHICS STATEMENT}

The studies involving human participants were reviewed and approved by the Ethics Committee of The Second Xiangya Hospital, Central South University [Ethical Audit No. Study 012 (2014)]. Written informed consent to participate in this study was provided by the participants' legal guardian/next of kin.

\section{AUTHOR CONTRIBUTIONS}

RZ and CW had primary responsibility for the protocol development, patient enrollment, data collecting, preliminary data analysis, and writing the manuscript. SW assisted with data analysis and critical revision for important content, and edited the draft. HC, FL, PL, and YW took the responsibility to complete the head-up tilt test and ambulatory blood pressure monitoring. CW supervised the design and execution of the study, checked the data analysis, and contributed to a final approval of the manuscript submitted. All authors have read and approved the final manuscript and assumed full responsibility for its contents.

\section{FUNDING}

This work was supported by grants from Hunan Province Clinical Medical Technology Innovation Guidance Project (2020SK53405) and Health and Family Planning Commission of Hunan Province in China (20201217).

Gong, S., Liu, K., Ye, R., Li, J., Yang, C., and Chen, X. (2019). Nocturnal dipping status and the association of morning blood pressure surge with subclinical target organ damage in untreated hypertensives. J. Clin. Hypertens 21, 12861294. doi: $10.1111 /$ jch. 13641

Grimaldi, Capitello, T., Fiorilli, C., Placidi, S., Vallone, R., Drago, F., et al. (2016). What factors influence parents' perception of the quality of life of children and adolescents with neurocardiogenic syncope? Health Qual. Life Outcomes 14:79. doi: 10.1186/s12955-016-0476-9

Holick, M. F., Binkley, N. C., Bischoff-Ferrari, H. A., Gordon, C. M., Heaney, R. P., et al. (2011). Evaluation, treatment, and prevention of vitamin D deficiency: an endocrine society clinical practice guideline. J. Clin. Endocrinol. Metab. 96, 1911-1930. doi: 10.1210/jc.2011-0385

Lahiri, M. K., Kannankeril, P. J., and Goldberger, J. J. (2008). Assessment of autonomic function in cardiovascular disease: physiological basis and prognostic implications. J. Am. Coll. Cardiol. 51, 1725-1733. doi: 10.1016/j.jacc. 2008.01.038

Latic, N., and Erben, R. G. (2020). Vitamin D and cardiovascular disease, with emphasis on hypertension, atherosclerosis, and heart failure. Int.J.Mol.Sci. 21, 6483. doi: 10.3390/ijms21186483

Mann, M. C., Exner, D. V., Hemmelgarn, B. R., Sola, D. Y., Turin, T. C., Ellis, L., et al. (2013). Vitamin D levels are associated with cardiac autonomic activity in healthy humans. Nutrients 5, 2114-2127. doi: 10.3390/nu5062114

Mann, M. C., Exner, D. V., Hemmelgarn, B. R., Turin, T. C., Sola, D. Y., Ellis, L., et al. (2014). Vitamin D supplementation is associated with improved 
modulation of cardiac autonomic tone in healthy humans. Int. J. Cardiol. 172, 506-508. doi: 10.1016/j.ijcard.2014.01.058

Medow, M. S., Stewart, J. M., Sanyal, S., Mumtaz, A., Sica, D., and Frishman, W. H. (2008). Pathophysiology, diagnosis, and treatment of orthostatic hypotension and vasovagal syncope. Cardiol. Rev. 16, 4-20. doi: 10.1097/CRD. 0b013e31815c8032

Naveilhan, P., Neveu, I., Baudet, C., Funakoshi, H., Wion, D., Brachet, P., et al. (1996). 1,25-Dihydroxyvitamin D3 regulates the expression of the low-affinity neurotrophin receptor. Brain Res. Mol. Brain Res. 41, 259-268. doi: 10.1016/ 0169-328x(96)00103-9

Saintonge, S., Bang, H., and Gerber, L. M. (2009). Implications of a new definition of vitamin $\mathrm{D}$ deficiency in a multiracial us adolescent population: the national health and nutrition examination survey III. Pediatrics 123, 797-803. doi: 10. 1542/peds.2008-1195

Stewart, J. M., Medow, M. S., Sutton, R., Visintainer, P., Jardine, D. L., and Wieling, W. (2017). Mechanisms of vasovagal syncope in the young: reduced systemic vascular resistance versus reduced cardiac output. J. Am. Heart Assoc. 6:e004417. doi: 10.1161/JAHA.116.004417

Tare, M., Emmett, S. J., Coleman, H. A., Skordilis, C., Eyles, D. W., Morley, R., et al. (2011). Vitamin D insufficiency is associated with impaired vascular endothelial and smooth muscle function and hypertension in young rats. J. Physiol. 589, 4777-4786. doi: 10.1113/jphysiol.2011.214726

Usalp, S., Kemal, H., Yuksek, U., Yaman, B., Gunsel, A., Edebal, O., et al. (2020). Is there any link between vitamin D deficiency and vasovagal syncope? J. Arrhythm. 36, 371-376. doi: 10.1002/joa3.12309

Villafane, J., Miller, J. R., Glickstein, J., Johnson, J. N., Wagner, J., Snyder, C. S., et al. (2021). Loss of consciousness in the young child. Pediatr. Cardiol. 42, 234-254. doi: 10.1007/s00246-020-02498-6

Wang, C., Li, Y., Liao, Y., Tian, H., Huang, M., Dong, X., et al. (2018). 2018 Chinese pediatric cardiology society (CPCS) guideline for diagnosis and treatment of syncope in children and adolescents. Sci. Bull. 63, 1558-1564. doi: 10.1016/j. scib.2018.09.019
Wang, Y., Zhang, C., Chen, S., Liu, P., Wang, Y., Tang, C., et al. (2019). Heart rate variability predicts therapeutic response to metoprolol in children with postural tachycardia syndrome. Front. Neurosci. 13:1214. doi: 10.3389/fnins.2019.0 1214

Williams, B., Mancia, G., Spiering, W., Agabiti, Rosei, E., Azizi, M., et al. (2018). 2018 ESC/ESH guidelines for the management of arterial hypertension. Eur. Heart J. 39, 3021-3104. doi: 10.1093/eurheartj/eh y339

Xie, H., Min, M., Guo, S., Xian, Yang, Yang, F., et al. (2020). Impact of vitamin $\mathrm{D}$ and vitamin $\mathrm{D}$ receptor on risk of cardiovascular diseases in children and adolescents with obesity in Sichuan, China: a cross-sectional study. Ann. Nutr. Metab. 76, 396-404. doi: 10.1159/000513287

Zhang, Q., Sun, Y., Zhang, C., Qi, J., and Du, J. (2021). Vitamin D deficiency and vasovagal syncope in children and adolescents. Front. Pediatr. 9:575923. doi: $10.3389 /$ fped.2021.575923

Conflict of Interest: The authors declare that the research was conducted in the absence of any commercial or financial relationships that could be construed as a potential conflict of interest.

Publisher's Note: All claims expressed in this article are solely those of the authors and do not necessarily represent those of their affiliated organizations, or those of the publisher, the editors and the reviewers. Any product that may be evaluated in this article, or claim that may be made by its manufacturer, is not guaranteed or endorsed by the publisher.

Copyright (c) 2021 Zou, Wang, Cai, Li, Lin, Wang and Wang. This is an open-access article distributed under the terms of the Creative Commons Attribution License (CC BY). The use, distribution or reproduction in other forums is permitted, provided the original author(s) and the copyright owner(s) are credited and that the original publication in this journal is cited, in accordance with accepted academic practice. No use, distribution or reproduction is permitted which does not comply with these terms. 